\title{
Delay-induced blow-up in a planar oscillation model
}

\author{
Alexey Eremin ${ }^{1} \cdot$ Emiko Ishiwata $^{2} \cdot$ Tetsuya Ishiwata $^{3} \cdot$ Yukihiko Nakata $^{4}$ \\ Received: 4 November 2020 / Revised: 11 May 2021 / Accepted: 22 May 2021 / \\ Published online: 22 July 2021 \\ (c) The Author(s) 2021
}

\begin{abstract}
In this paper we study a system of delay differential equations from the viewpoint of a finite time blow-up of the solution. We prove that the system admits blow-up solutions, no matter how small the length of the delay is. In the non-delay system every solution approaches to a stable unit circle in the plane, thus time delay induces blowup of solutions, which we call "delay-induced blow-up" phenomenon. Furthermore, it is shown that the system has a family of infinitely many periodic solutions, while the non-delay system has only one stable limit cycle. The system studied in this paper is an example that arbitrary small delay can be responsible for a drastic change of the dynamics. We show numerical examples to illustrate our theoretical results.
\end{abstract}

Keywords Blow-up of solutions · Periodic solutions $\cdot$ Delay differential equations

Mathematics Subject Classification 34K99 · 34K13

\section{Introduction}

In various disciplines of the science, mathematical modelling offers a description of phenomena. In some phenomena, the history of the state, not only the current state, affects the change of the state, thus it is reasonable to consider the effect of time delay [7]. Up to now the theory of delay differential equations have been intensively developed [9, 17].

\footnotetext{
This work was supported by KAKENHI Nos. 26400212, 15K13461, 19H05599, 19K21836 and $20 \mathrm{~K} 03734$.

Tetsuya Ishiwata

tisiwata@shibaura-it.ac.jp

1 Department of Information Systems, Saint-Petersburg State University, St. Petersburg, Russia

2 Department of Applied Mathematics, Tokyo University of Science, Tokyo, Japan

3 Department of Mathematical Sciences, Shibaura Institute of Technology, Tokyo, Japan

4 Department of Mathematical Sciences, Aoyama Gakuin University, Tokyo, Japan
} 
In this paper we study a system of delay differential equations from the viewpoint of the blow-up solutions. Here we use the terminology "blow-up" as a finite time blow-up of the solutions, i.e., the solution diverges (in a suitable topology) in finite time. The blow-up phenomenon has been widely investigated in partial differential equations, see $[2,11,18,19]$ and references therein. There are also extensive studies in Volterra integral equation, as an alternative formulation of partial differential equation of parabolic type with a point source term [3, 18, 21]. Compactification of the phase space is a method to study the blow-up solutions of ordinary differential equations $[6,14]$. Numerical analysis has been an unavoidable tool for understanding the blow-up phenomenon. Numerical method to compute the blow-up solutions for polynomial systems of ordinary differential equations has been proposed for ordinary differential equations with application to partial differential equations, see [11] and references therein. Recently, numerical validation for the existence of the blow-up solutions is proposed [20].

The blow-up phenomenon in delay differential equations has not been much studied, as far as we know, except for a few studies [1, 8, 10, 13, 22, 23]. Perhaps the reason is that many examples of delay differential equations, which appear in population biology, control theory, etc, negative feedback condition is usually imposed which excludes blow-up solutions. One also sees that the following delay differential equation

$$
x^{\prime}(t)=x(t) x(t-\tau)
$$

does not have a blow-up solution (at least if the initial function is continuous) for $\tau>0$ and is reduced to a famous example of the ordinary differential equation having a blow-up solution when $\tau=0$. Thus one may speculate that time delay inhibits the blow-up phenomenon in general (which is certainly not).

In the paper [8] the authors study the existence of the blow-up solutions for a class of delay differential equations. The authors are interested if adding (and multiplying) a delay term to an ordinary differential equation affects the solution behavior. Using the comparison principle, the authors obtain conditions that the delay term does not change the qualitative properties concerning the global existence and blow-up of the solution. In [23] the authors study the blow-up phenomenon of differential equations with piecewise constant arguments, in comparison with the corresponding ordinary differential equations. The blow-up phenomenon is studied in Volterra integro-differential equations. See $[1,10,13,22]$ as applications to a parabolic type partial differential equations to study of Volterra integro-differential equations.

It seems that the blow-up phenomenon that stems from the time delay has not been reported, to the best of our knowledge. Our motivations are to demonstrate whether the time delay itself induces blow-up of the solution, and to understand the mechanism of blow-up of solutions. In this paper, we propose an example model that time delay drastically changes the solution behavior and induces the blow-up solution together with a family of infinitely many periodic solutions, where most of solutions are shown to be unstable. In our equation, a blow-up solution exists no matter how small the length of the delay is, while non-delay equation does not have a blow-up solution. We thus call these phenomena "delay-induced blow-up". 
This paper is organized as follows. In Sect. 2, we introduce a planar system of delay differential equations which we study in this paper. In the absence of time delay, the system becomes a planar system of ordinary differential equations. It can be seen that every solution except the trivial solution approaches to the limit cycle, thus no solution blows up. Concerning the system of delay differential equations, we present our two main theorems that show blow-up of solutions is possible due to time delay and that the system admits infinitely many periodic solutions. In this section we use a special initial function in order to show blow-up of solutions for any $\tau>0$. We demonstrate numerical examples of blow-up solutions and global solutions. See Remark 3.1, Fig. 1 and also Sect. 6. In Sect. 3, by a careful estimation of the solution we show that there is a blow-up solution for the system of delay differential equations and provide a proof of Theorem 2.1. In Sect. 4, we study existence of a periodic solution with constant radius and constant angular velocity in the plane. It is shown that there exist infinitely many periodic solutions which appear due to time delay. In Sect. 5, we study a characteristic equation which characterizes stability of the periodic solutions. It is shown that most of periodic solutions are not stable except for the only one periodic solution that is a continuation of the periodic solution of the non-delay model. In Sect. 6, we provide numerical examples which illustrate our theoretical results. In Sect. 7, we discuss our results.

\section{A planar system of delay differential equations and main results}

Let $\tau \geq 0$ be a parameter for time delay. In this paper we consider the following planar system of delay differential equations

$$
\begin{aligned}
& x^{\prime}(t)=x(t)-y(t)-x(t-\tau)\left(x^{2}(t)+y^{2}(t)\right), \\
& y^{\prime}(t)=x(t)+y(t)-y(t-\tau)\left(x^{2}(t)+y^{2}(t)\right) .
\end{aligned}
$$

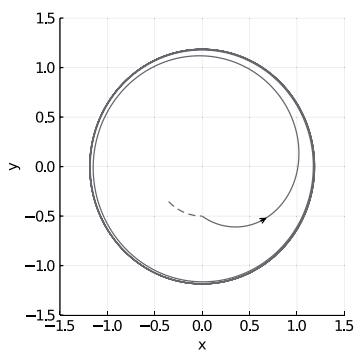

(i)

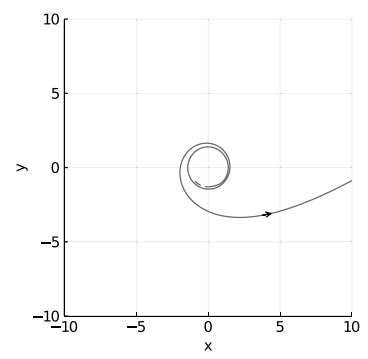

(ii)

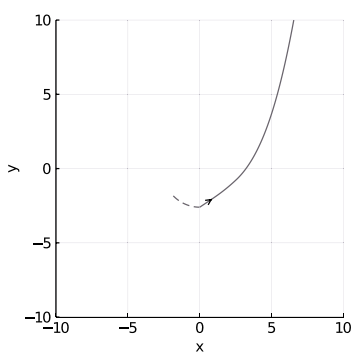

(iii)

Fig. 1 Illustration of trajectories of the solution in $(x, y)$-plane for $\tau=0.392$. The initial condition (3.2), which are plotted as dashed curves in the above figures, is used (in the polar coordinate) with $s(t)=\frac{\pi}{2 \tau} t-\frac{1}{2} \pi$ for the numerical experiments. The solution behavior changes by different $R=0.5,1.3$ and 2.6. (i) The solution converges to a periodic solution with $R=0.5$, (ii) The solution blows up in a finite time $t>\tau$ with $R=1.3$ (The blow-up time is nearly $t=4.359 \cdots$.) and (iii) The solution blows up in $t<\tau$ with $R=2.6$ 
For the special case $\tau=0$, the system (2.1) is reduced to the following system of ordinary differential equations with $a=1$ :

$$
\begin{aligned}
& x^{\prime}(t)=a x(t)-y(t)-x(t)\left(x^{2}(t)+y^{2}(t)\right), \\
& y^{\prime}(t)=x(t)+a y(t)-y(t)\left(x^{2}(t)+y^{2}(t)\right) .
\end{aligned}
$$

Here $a \in \mathbb{R}$ is a parameter. This system is a famous model for Hopf bifurcation at $a=0$. (See [12], for instance.) From the elementary calculation, one can see that every solution of (2.2) except the trivial solution $(x(t), y(t)) \equiv(0,0)$ tends to a periodic solution of minimal period $2 \pi$ and satisfies

$$
\lim _{t \rightarrow \infty} \sqrt{x^{2}(t)+y^{2}(t)}=1 .
$$

i.e., the limit cycle of (2.2) is the unit circle.

For the system (2.1) we prove that the system admits blow-up solutions due to the presence of time delay. We prove the following theorem in Sect. 3.

Theorem 2.1 For any $\tau>0$, there exist blow-up solutions for the planar system of delay differential Eq. (2.1).

Then in Sects. 4 and 5, we further investigate the system (2.1) and show the existence of infinitely many periodic solutions. We also study the stability of the periodic solutions. The following theorem is proved in Sects. 4 and 5.

Theorem 2.2 For any $\tau>0$, there exist infinitely many unstable periodic solutions of (2.1) with constant radius and angular velocity. Moreover, there exists a positive $\tau^{*}$ such that the system (2.1) admits only one asymptotically stable periodic solution with constant radius and angular velocity for $0<\tau<\tau^{*}$.

\section{Delay-induced blow-up}

We consider a polar coordinate system. Let

$$
(x(t), y(t))=r(t)(\cos \theta(t), \sin \theta(t)) .
$$

By the change of the variables, from (2.1), we obtain the following polar coordinate system

$$
\begin{gathered}
r^{\prime}(t)=r(t)(1-r(t) r(t-\tau) \cos (\theta(t)-\theta(t-\tau))), \\
\theta^{\prime}(t)=1+r(t) r(t-\tau) \sin (\theta(t)-\theta(t-\tau)) .
\end{gathered}
$$

Now we prove that there exists a solution such that $r$ blows up in a finite time with $\theta \rightarrow \frac{\pi}{4}$ as $r \rightarrow \infty$. Therefore, we can conclude that $x$ and $y$ blow up in a finite time. 
For (3.1), we consider the following initial condition

$$
\begin{gathered}
r(t)=R, t \in[-\tau, 0], \\
\theta(t)= \begin{cases}-\frac{3}{4} \pi, & -\tau \leq t<-\frac{1}{2} \tau, \\
s(t), & -\frac{1}{2} \tau \leq t \leq 0,\end{cases}
\end{gathered}
$$

where $R>0$ and $s(t)$ is a continuous function such that

$$
s\left(-\frac{1}{2} \tau\right)=-\frac{3}{4} \pi, s(0)=-\frac{1}{2} \pi .
$$

Remark 3.1 In this paper we consider a special initial condition (3.2) in order to show an existence of blow-up solutions for any positive $\tau$. In this section we will prove the blow-up of solutions in $t<\tau$ for sufficiently large $R>0$. For other initial data, there are no mathematical results on global existence of solutions except periodic solutions studied in Sect. 4 and blow-up of the solutions. In Fig. 1 (i) and (ii), numerical examples for $R=0.5$ and 1.3 are presented. These numerical simulations suggest that there are not only time-global solutions which converge to a periodic orbit but also solutions which blow up in a finite time $t>\tau$. In Sect. 6, numerical figures for other initial data are presented. have

Since $-\tau \leq t-\tau<-\frac{1}{2} \tau$ for $t \in\left[0, \frac{1}{2} \tau\right]$, from the initial condition (3.2), we

$$
r(t-\tau)=R, \theta(t-\tau)=-\frac{3}{4} \pi, t \in\left[0, \frac{1}{2} \tau\right] .
$$

Then, for $t \in\left[0, \frac{1}{2} \tau\right]$, the solution of the system of delay differential equations (3.1) with the initial condition (3.2) is given by the following system of ordinary differential equations

$$
\begin{gathered}
r^{\prime}(t)=r(t)\left(1-R r(t) \cos \left(\theta(t)+\frac{3}{4} \pi\right)\right), \\
\theta^{\prime}(t)=1+R r(t) \sin \left(\theta(t)+\frac{3}{4} \pi\right)
\end{gathered}
$$

with the initial condition

$$
(r(0), \theta(0))=\left(R,-\frac{1}{2} \pi\right)
$$

We are going to prove that the solution of the system of ordinary differential equations (3.3) with the initial condition (3.4) blows up in $t \leq \frac{1}{2} \tau$.

Figure 2 shows each step for blow-up of solutions. The process for blow-up of solutions is divided into the following 3 steps: 
Step 1 The angle of the solutions is monotonically increasing from $-\pi / 2$ to $-\pi / 4$ in $t \in\left[0, T_{1}\right]$ for some $T_{1}>0$. In this region, the radius of the solutions may decrease, and thus we establish a decay estimate of the solutions in Lemma 3.1.

Step 2 Both radius and angle of the solutions monotonically increase in $t \in\left[T_{1}, T_{2}\right]$ for some $T_{2}>T_{1}$. The angle varies from $-\pi / 4$ to 0 and the radius grows up beyond a threshold for an emergence of a nullcline of the angle. (See Lemmas 3.2, 3.3.)

Step 3 The final stage for blow-up of solutions. The angle monotonically reaches to the nullcline of the angle which appears in Step 2. Then we show in Lemma 3.4 that for large $R$ there exists a blow-up time $T_{3}\left(T_{2}<T_{3}\right)$ with $T_{3}<\frac{1}{2} \tau$ such that

$$
\lim _{t \uparrow T_{3}} r(t)=\infty .
$$

For the exposition, we define

$$
\phi(t):=\theta(t)+\frac{3}{4} \pi, t \geq 0 .
$$

Then the system (3.3) with the initial condition (3.4) becomes

$$
r^{\prime}(t)=r(t)(1-\operatorname{Rr}(t) \cos \phi(t)),
$$

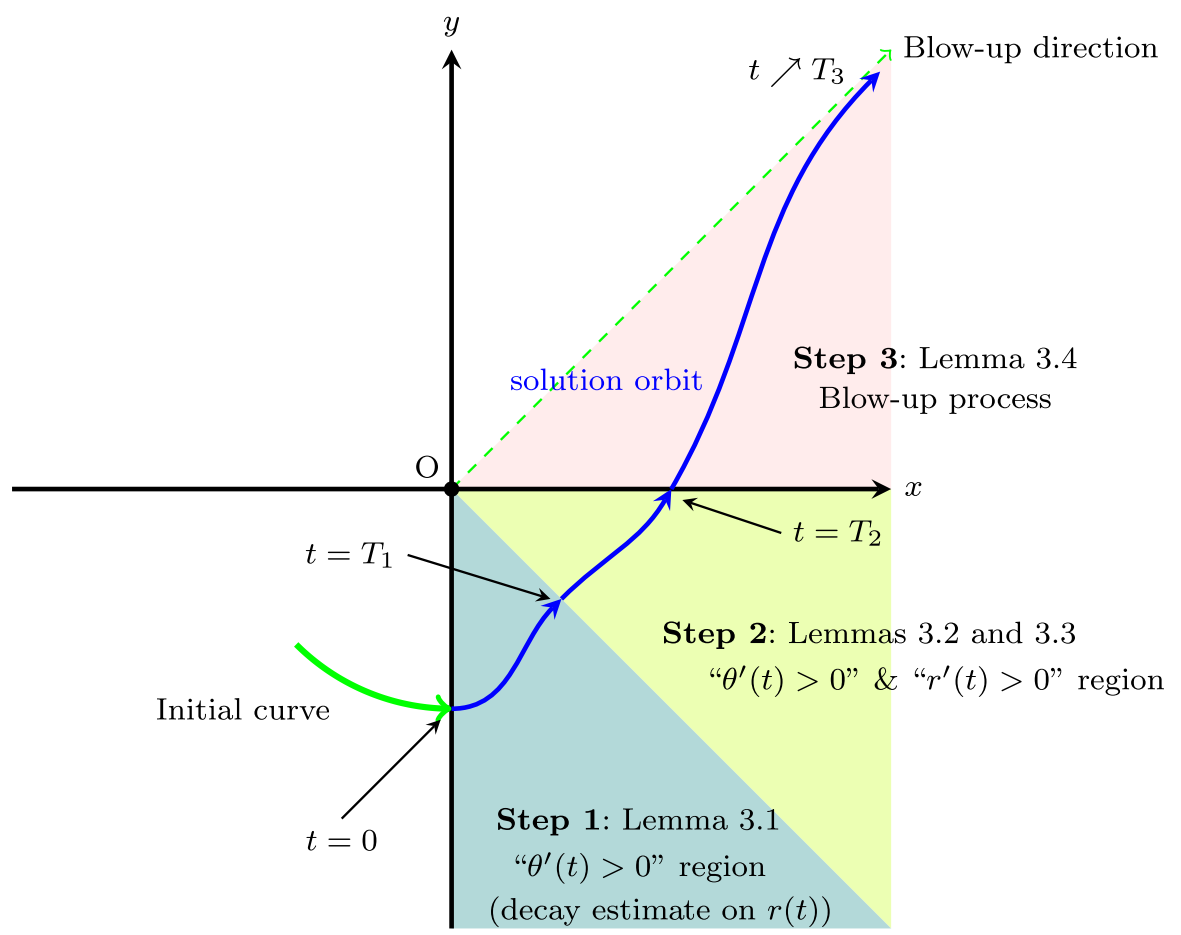

Fig. 2 Three steps for the blow-up of solutions 


$$
\phi^{\prime}(t)=1+\operatorname{Rr}(t) \sin \phi(t)
$$

with the initial condition

$$
(r(0), \phi(0))=\left(R, \frac{1}{4} \pi\right)
$$

In the proof below we need to estimate the solution $r$. For the estimation we use the following equation

$$
\frac{d r}{d \phi}=\frac{r(1-\operatorname{Rr} \cos \phi)}{1+R r \sin \phi},
$$

which is obtained from (3.6).

\subsection{Step 1: The decay estimate of the radius}

Lemma 3.1 There exists $T_{1}>0$ such that $\phi$ monotonically increases from $\pi / 4$ to $\pi / 2$ for $t \in\left[0, T_{1}\right]$. One also has

$$
R \exp \left(-\left(\phi(t)-\frac{\pi}{4}\right)\right) \leq r(t) \leq R \exp \left(\phi(t)-\frac{\pi}{4}\right), t \in\left[0, T_{1}\right]
$$

Moreover, there exists $\bar{R}_{1}>0$ such that

$$
R>\bar{R}_{1} \Longrightarrow T_{1}<\frac{\tau}{4}
$$

Proof The solution of the system of ordinary differential equation (3.6) with the initial condition (3.7) exists for sufficiently small $t$. We show that there exists $T_{1}>0$ such that the solution exists for $t \in\left[0, T_{1}\right]$ satisfying that $\phi$ monotonically increases from $\pi / 4$ to $\pi / 2$ for $0 \leq t \leq T_{1}$. We first obtain an a priori estimate for $r$ for $\phi \in\left[\frac{1}{4} \pi, \frac{1}{2} \pi\right]$. From (3.8) it follows that

$$
\frac{d r}{d \phi} \leq r, \phi \in\left[\frac{1}{4} \pi, \frac{1}{2} \pi\right]
$$

One also obtains the following estimation

$$
\frac{d r}{d \phi} \geq \frac{-R r^{2} \cos \phi}{R r \sin \phi} \geq \frac{-R r^{2} \frac{\sqrt{2}}{2}}{R r \frac{\sqrt{2}}{2}}=-r, \phi \in\left[\frac{1}{4} \pi, \frac{1}{2} \pi\right] .
$$

Integrating the inequalities (3.10) and (3.11), we obtain the following estimation

$$
R \exp \left(-\left(\phi-\frac{\pi}{4}\right)\right) \leq r \leq R \exp \left(\phi-\frac{\pi}{4}\right), \phi \in\left[\frac{1}{4} \pi, \frac{1}{2} \pi\right] .
$$


Using the a priori bound (3.12), from the equation (3.6b), we see that $\phi$ is an increasing function and that $\phi^{\prime}(t) \geq 1$, provided $\phi \in\left[\frac{1}{4} \pi, \frac{1}{2} \pi\right]$. Therefore, there exists $T_{1}>0$ such that $\phi$ monotonically increases from $\pi / 4$ to $\pi / 2$ for $t \in\left[0, T_{1}\right]$. The inequality (3.9) holds from the estimation (3.12). Since, from the inequality (3.9), we have $R \exp (-\pi / 4) \leq r$ for $t \in\left[0, T_{1}\right]$, the following estimation

$$
\phi^{\prime(t) \geq 1+R^{2} \exp \left(-\frac{\pi}{4}\right)} \sin \phi \geq 1+R^{2} \exp \left(-\frac{\pi}{4}\right) \frac{\sqrt{2}}{2}, t \in\left[0, T_{1}\right]
$$

implies that there exists $\bar{R}_{1}>0$ such that if $R>\bar{R}_{1}$ then $T_{1}<\frac{\tau}{4}$.

From Lemma 3.1, we have

$$
\begin{aligned}
& R \exp \left(-\frac{\pi}{4}\right) \leq r\left(T_{1}\right) \leq R \exp \left(\frac{\pi}{4}\right), \\
& \phi\left(T_{1}\right)=\frac{1}{2} \pi .
\end{aligned}
$$

\subsection{Step 2: Emergence of the nullcline of the angle}

Next we have the following estimation.

Lemma 3.2 There exists $T_{2}\left(>T_{1}\right)$ such that $\phi$ monotonically increases from $\pi / 2$ to $3 \pi / 4$ for $t \in\left[T_{1}, T_{2}\right]$. One also has that $r$ monotonically increases for $t \in\left[T_{1}, T_{2}\right]$ and

$$
R \exp \left(-\frac{\pi}{4}\right) \leq r(t)<\infty, t \in\left[T_{1}, T_{2}\right]
$$

Moreover, there exists $\bar{R}_{2}>0$ such that

$$
R>\bar{R}_{2} \Longrightarrow T_{2}-T_{1}<\frac{\tau}{8}
$$

Proof First, we derive an a priori estimate for $r$, provided $\phi \in\left[\frac{1}{2} \pi, \frac{3}{4} \pi\right]$. Let $\phi \in\left[\frac{1}{2} \pi, \frac{3}{4} \pi\right]$. We have $r^{\prime}(t) \geq r$ from (3.6a), thus $r$ monotonically increases. Then one has

$$
r \geq r\left(T_{1}\right) \geq R \exp \left(-\frac{\pi}{4}\right)
$$

Since, from (3.8), it holds that

$$
\frac{d r}{d \phi} \leq \frac{r\left(1+R r \frac{\sqrt{2}}{2}\right)}{R r \frac{\sqrt{2}}{2}}=\frac{\sqrt{2}+R r}{R}, \phi \in\left[\frac{1}{2} \pi, \frac{3}{4} \pi\right],
$$


integrating the equation, we obtain

$$
\log \left(\frac{\sqrt{2}+R r}{\sqrt{2}+\operatorname{Rr}\left(T_{1}\right)}\right) \leq \phi-\frac{\pi}{2},
$$

which implies $r<\infty$.

From the Eq. (3.6b) and the a priori bounds (3.15), we have that $\phi^{\prime}(t) \geq 1$ holds for $\phi \in\left[\frac{1}{2} \pi, \frac{3}{4} \pi\right]$. Hence, there exists $T_{2}>T_{1}$ such that $\phi$ monotonically increases for $t \in\left[T_{1}, T_{2}\right]$ with $\phi\left(T_{1}\right)=\frac{1}{2} \pi, \phi(t) \in\left(\frac{1}{2} \pi, \frac{3}{4} \pi\right)$ for $t \in\left(T_{1}, T_{2}\right)$ and $\phi\left(T_{2}\right)=\frac{3}{4} \pi$. Therefore, the lower estimate (3.15) is valid for $t \in\left[T_{1}, T_{2}\right]$ and by virtue of (3.17) the boundedness of $r(t)$ also holds for $t \in\left[T_{1}, T_{2}\right]$. That is, the inequality (3.14) holds for $t \in\left[T_{1}, T_{2}\right]$. Finally, from the inequality (3.14), the following estimation holds:

$$
\phi^{\prime}(t) \geq 1+R^{2} \exp \left(-\frac{\pi}{4}\right) \sin \phi \geq 1+R^{2} \exp \left(-\frac{\pi}{4}\right) \frac{\sqrt{2}}{2}, t \in\left[T_{1}, T_{2}\right],
$$

which implies that there exists $\bar{R}_{2}>0$ such that if $R>\bar{R}_{2}$ then $T_{2}-T_{1}<\frac{\tau}{8}$.

We are ready to show that the solution of (3.6) blows up. Note that $r^{\prime}\left(T_{2}\right) \geq r\left(T_{2}\right)>0 \quad$ and $\phi^{\prime}\left(T_{2}\right)>1$ since $\cos \phi\left(T_{2}\right)=\cos \frac{3}{4} \pi=-\frac{\sqrt{2}}{2}<0 \quad$ and $\sin \phi\left(T_{2}\right)=\sin \frac{3}{4} \pi=\frac{\sqrt{2}}{2}>0$. Thus, there is sufficiently small $\delta>0$ such that for $t \in\left(T_{2}, T_{2}+\delta\right)$ the solution exists and that $\phi$ and $r$ increase and thus $r(t) \geq r\left(T_{2}\right) \geq R \exp \left(-\frac{\pi}{4}\right)$ for $t \in\left(T_{2}, T_{2}+\delta\right)$.

In Fig. 3, we plot the graph of $\left(\phi, \phi^{\prime}\right)$. We show that, for sufficiently large $R$, a nullcline for $\phi$ exists in $\left(\pi, \frac{3}{2} \pi\right)$, where the right-hand side of the $\phi$-equation (3.6b) becomes 0 . We see in Lemma 3.4 that $\phi$ has a upper bound for suitable large $R$ and then $r(t)$ blows up in a finite time. We now let $R$ be a sufficiently large number such that $R^{2} \exp \left(-\frac{\pi}{4}\right)>\sqrt{2}$. Then, for $r>R \exp \left(-\frac{\pi}{4}\right)$, there is a $\phi$-nullcline in $\left(\pi, \frac{5}{4} \pi\right)$ that is given as

$$
\phi^{*}(r):=\pi-\arcsin \left(-\frac{1}{R r}\right) \in\left(\pi, \frac{5}{4} \pi\right), r>R \exp \left(-\frac{\pi}{4}\right) .
$$

It is easy to obtain the following elementary lemma.

Lemma 3.3 Let $R$ be a sufficiently large number such that $R^{2} \exp \left(-\frac{\pi}{4}\right)>\sqrt{2}$. Then $\phi^{*}(r) \in\left(\pi, \frac{5}{4} \pi\right)\left(r>R \exp \left(-\frac{\pi}{4}\right)\right)$. One has that

$$
1+\operatorname{Rr} \sin \phi\left\{\begin{array}{l}
>0, \phi \in\left(\frac{3}{4} \pi, \phi^{*}(r)\right) \\
=0, \phi=\phi^{*}(r)
\end{array}\right.
$$

and that 


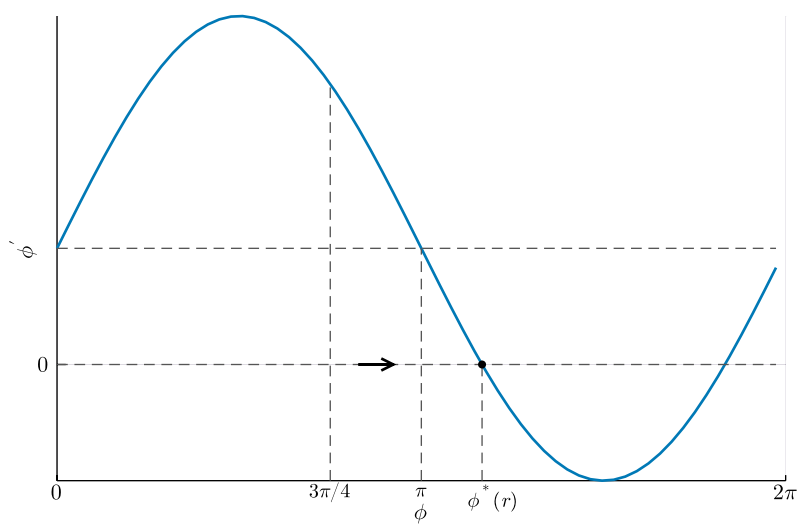

Fig. 3 The graph of the right-hand side of the equation (3.6b) for large $R r$. Large $R r$ makes a nullcline $\phi^{*}(r)$ of the Eq. (3.6b). $\phi$ approaches to $\phi^{*}(r)$

$$
\lim _{r \rightarrow \infty} \phi^{*}(r)=\pi
$$

Hence, as long as the solution exists, for $t>T_{2}, r(t) \geq r\left(T_{2}\right) \geq R \exp \left(-\frac{\pi}{4}\right)$ increases and $\phi$ increases and tends to $\phi^{*}(r(t))$. Observe that $\phi$ stays in the interval $\left(\frac{3}{4} \pi, \frac{5}{4} \pi\right)$, thus the sign of $\cos \phi$ in the right hand side of (3.6a) is fixed and is positive.

From Lemma 3.2, we have

$$
\begin{aligned}
& R \exp \left(-\frac{\pi}{4}\right) \leq r\left(T_{2}\right)<\infty, \\
& \phi\left(T_{2}\right)=\frac{3}{4} \pi
\end{aligned}
$$

\subsection{Step 3: Blow-up of solutions}

Finally we show the blow-up of solutions.

Lemma 3.4 There exists $T_{3}\left(>T_{2}\right)$ such that $\phi$ and $r$ monotonically increase for $t \in\left[T_{2}, T_{3}\right)$ and

$$
\lim _{t \uparrow T_{3}} r(t)=\infty, \lim _{t \uparrow T_{3}} \phi(t)=\pi .
$$

Furthermore, there exists $\bar{R}_{3}>0$ such that

$$
R>\bar{R}_{3} \Longrightarrow T_{3}-T_{2}<\frac{\tau}{8} \text {. }
$$


Proof We consider the system (3.6) for $t>T_{2}$. Let $R$ be a sufficiently large number such that $R>\max \left\{\bar{R}_{1}, \bar{R}_{2}\right\}$ and that $R^{2} \exp \left(-\frac{\pi}{4}\right)>\sqrt{2}$. Since $R>\max \left\{\bar{R}_{1}, \bar{R}_{2}\right\}$, from Lemmas 3.1 and 3.2, we have $\phi\left(T_{2}\right)=\frac{3}{4} \pi$ and $T_{2}<\frac{3}{8} \tau$. We derive an a priori estimate for $\phi$, provided $r<\infty$. Since we have $R^{2} \exp \left(-\frac{\pi}{4}\right)>\sqrt{2}$, one sees that the equation (3.6b) has an equilibrium, $\phi^{*}(r) \in\left(\pi, \frac{5}{4} \pi\right)$ from Lemma 3.3. Suppose that there exists $t^{*}>T_{2}$ such that $\phi^{\prime}\left(t^{*}\right)=0$ while $r\left(t^{*}\right)<\infty$. Note that $\phi\left(t^{*}\right) \in\left(\pi, \frac{5}{4} \pi\right)$ and by (3.8) we have $\left.\frac{d r}{d \phi}\right|_{t=t^{*}}=\infty$, that is, the solution orbit crosses the curve of $\phi$ -nullcline $\left\{(\phi, r) \mid 1+R r \sin \phi=0, \pi<\phi<\frac{5}{4} \pi\right\}$ vertically. Then, the solution enters the region where $\phi^{\prime}(t)<0$, in what follows $\phi(t)<\frac{5}{4} \pi$. Note that the intersection of the solution orbit and $\phi$-nullcline may occur at most once because of the shape of the nullcline and the fact of vertical crossing. See Fig. 4.

One sees that $\phi(t) \in\left(\frac{3}{4} \pi, \frac{5}{4} \pi\right)$. Since

$$
-1 \leq \cos \phi \leq-\frac{\sqrt{2}}{2}, \phi \in\left(\frac{3}{4} \pi, \frac{5}{4} \pi\right),
$$

from (3.6a) we now have the estimation,

$$
r^{\prime}(t) \geq r\left(1+R \frac{\sqrt{2}}{2} r\right) .
$$

Therefore, one sees that there exists $T_{3}$ such that $\lim _{t \uparrow T_{3}} r(t)=\infty$. Thus, from (3.19) in Lemma 3.3, we have $\lim _{t \uparrow T_{3}} \phi(t)=\frac{\pi}{2}$. From the Eq. (3.21), for any $\underline{\varepsilon}$ there exists $\bar{R}$ such that $R>\bar{R}$ implies that $T_{3}-T_{2}^{2}<\varepsilon$. Therefore, there exists $\bar{R}_{3}$, such that $T_{3}-T_{2}<\frac{\tau}{8}$.

Proof of Theorem 2.1 Let $R>\bar{R}_{3}$. From Lemmas 3.1, 3.2 and 3.4, one has $T_{3}<\frac{\tau}{2}$. (3.20) in Lemma 3.4 implies that

$$
\lim _{t \uparrow T_{3}}(x(t), y(t))=(\infty, \infty) .
$$

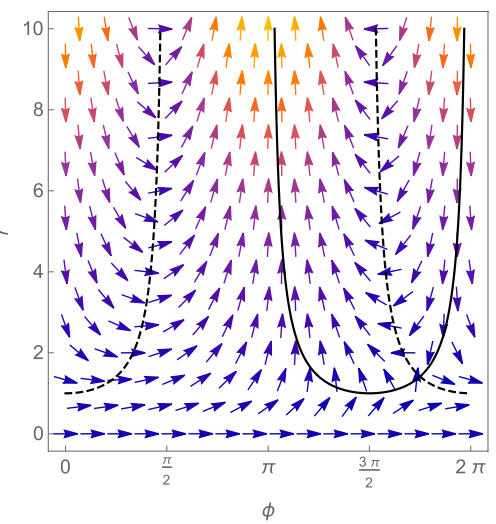

Fig. 4 The vector field $\left(r^{\prime}(t), \phi^{\prime}(t)\right)$ for the system (3.6). Solid lines (resp. dashed lines) describe $\phi$ -nullcline (resp. $r$-nullcline) 
Thus we obtain the conclusion.

\section{Existence of periodic solutions}

We consider a periodic solution with a constant radius, i.e. $r(t) \equiv \rho>0$, for the system (3.1). Then, $\theta^{\prime}(t)$ is also a constant from Eq. (3.1). Thus, we treat a periodic solution of the form

$$
(r(t), \theta(t))=(\rho, \omega t),
$$

where $\omega \in \mathbb{R}$ is an angular velocity.

From (3.1), the periodic solution satisfies

$$
\begin{gathered}
1=\rho^{2} \cos \omega \tau, \\
\omega=1+\rho^{2} \sin \omega \tau .
\end{gathered}
$$

Remark that for the special case $\tau=0$, from (4.2), we obtain

$$
(\rho, \omega)=(1,1),
$$

for periodic solution $(r(t), \theta(t))=(1, t)$ corresponding to the system (3.1) with $\tau=0$.

From (4.2) one has $\cos \omega \tau>0$. Then we have the following equations

$$
\begin{gathered}
\omega-1=\tan \omega \tau, \\
1+(\omega-1)^{2}=\rho^{4} .
\end{gathered}
$$

In Fig. 5a we plot the functions $y=\omega-1$ and $y=\tan \omega \tau$. For $\omega \tau \in\left(-\frac{\pi}{2}+2 n \pi, \frac{\pi}{2}+2 n \pi\right)(n \in \mathbb{Z})$, intersections of the two functions correspond to the roots of (4.4a) satisfying $\cos \omega \tau>0$. First we study the roots of (4.4a) for $0 \leq \omega \tau<\frac{\pi}{2}$. The implicit function (4.4a) for $0 \leq \omega \tau<\frac{\pi}{2}$ defines a function

$$
\bar{\tau}(\omega)=\frac{\arctan (\omega-1)}{\omega},
$$

which attains a unique maximum at $\omega=\omega^{*}$ where $\bar{\tau}^{\prime}\left(\omega^{*}\right)=0$. One can compute that

$$
\bar{\tau}^{\prime}(\omega)=\frac{\frac{\omega}{1+(\omega-1)^{2}}-\arctan (\omega-1)}{\omega^{2}},
$$

from which we can numerically compute $\omega^{*} \approx 2.22913 \cdots$. Let $\tau^{*}=\bar{\tau}\left(\omega^{*}\right)$. Then we numerically obtain

$$
\tau^{*} \approx 0.398284 \cdots .
$$

Proposition 4.1 For $0<\tau \leq \tau^{*}$ the equation (4.2) has two roots which we denote by $\omega_{0}(\tau)$ and $\omega_{1}(\tau)$ such that 


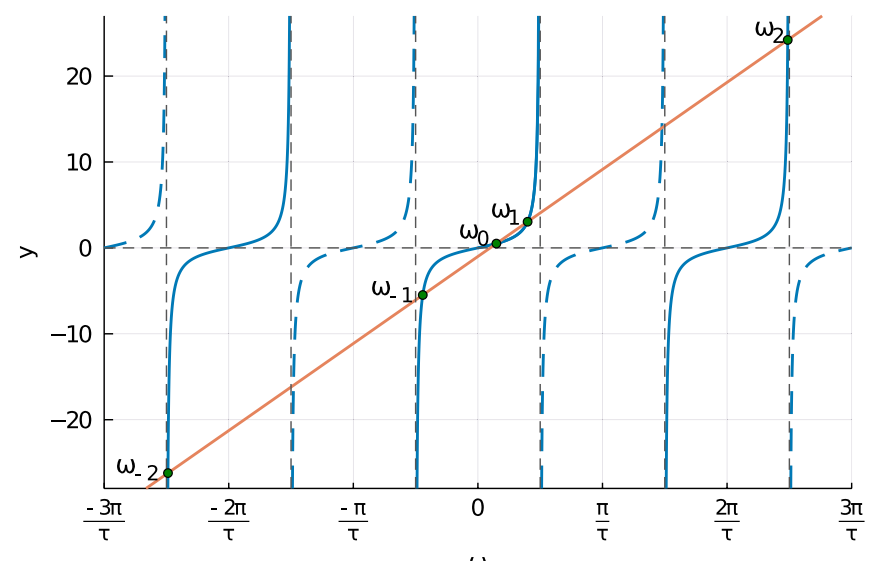

$\omega$

(a) The roots of the equation (4.4a) are given as intersections of two graphs $y=\omega-1$ and $y=\tan (\omega \tau)$.

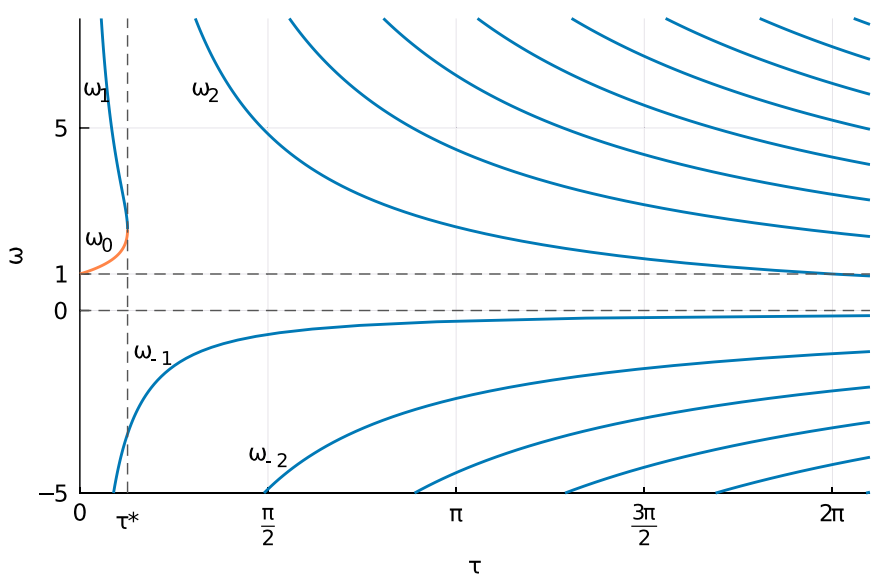

(b) Branches of the periodic solutions in $(\tau, \omega)$ plane. The branch $\omega_{0}$ emerges from $\omega=1$ at $\tau=0$ and the branch $\omega_{1}$ emerges from $\omega=+\infty$ at $\tau=0$. Those branches $\omega_{0}$ and $\omega_{1}$ meet at $\tau=\tau^{*}$. The branches $\omega_{j} j \in \mathbb{Z} \backslash\{0\}$ appear from either $+\infty$ or $-\infty$ at $\tau=0$.

Fig. 5 Visualization of solutions of equation (4.4a)

- $\omega_{0}(\tau) \leq \omega^{*} \leq \omega_{1}(\tau)$,

- $\lim _{\tau \downarrow 0} \omega_{0}(\tau)=1, \lim _{\tau \downarrow 0} \omega_{1}(\tau)=\infty$ and

- $\omega_{0}\left(\tau^{*}\right)=\omega_{1}\left(\tau^{*}\right)=\omega^{*}$.

For $\tau<\tau^{*}$ there is at least 2 periodic solutions

$$
(r(t), \theta(t))=\left(\rho_{j}, \omega_{j} t\right), \quad j \in\{0,1\},
$$

where 


$$
\rho_{j}:=\left(1+\left(\omega_{j}-1\right)^{2}\right)^{\frac{1}{4}}
$$

From Proposition 4.1 and (4.6), one sees that $\lim _{\tau \downarrow 0} \rho_{0}=1$ and $\lim _{\tau \downarrow 0} \rho_{1}=\infty$. Thus one periodic solution is a continuation of the periodic solution (4.3) at $\tau=0$ and one periodic solution emerges from infinity.

For $\tau>0$, one can see that the Eq. (4.2) has infinitely many roots. It is elementary to prove the following result, thus we omit the proof. See also Fig. 5a.

Lemma 4.1 For $j \in \mathbb{Z} \backslash\{0,1\}$, the Eq. (4.4a) with $\cos \omega \tau>0$ has exactly one root $\omega_{j}$ on the following each interval

$$
\begin{array}{ll}
\omega_{j} \in\left(\frac{2 \pi}{\tau}(j-1), \frac{2 \pi}{\tau}(j-1)+\frac{\pi}{2 \tau}\right), & j=2,3, \ldots, \\
\omega_{j} \in\left(\frac{2 \pi}{\tau}(j+1)-\frac{\pi}{2 \tau}, \frac{2 \pi}{\tau}(j+1)\right), & j=\ldots,-2,-1 .
\end{array}
$$

Therefore, we obtain roots $\omega_{j}$ for $j \in \mathbb{Z}$ if $\tau \leq \tau^{*}$ and for $j \in \mathbb{Z} \backslash\{0,1\}$ if $\tau>\tau^{*}$ of the Eq. (4.4a). In Fig. 5b we plot the branches for $\omega$ as a function of $\tau$. Once $\omega$ of the periodic solution (4.1) is given, $\rho$ is determined from (4.4b). The radius $\rho$ can be determined as $\rho_{j}$ given as, similar to (4.6),

$$
\rho_{j}=\left(1+\left(\omega_{j}-1\right)^{2}\right)^{\frac{1}{4}} \geq 1
$$

This implies that the periodic solutions of the form (4.1) has larger radius than the unit circle which is the trajectory of the periodic solution for $\tau=0$. We also note that there is a root $\rho=1$ if $\tau=2 n \pi, n \in \mathbb{N}$. In Fig. 6 we plot the branches for the radius as a function of $\tau$.

Summarizing the above findings, we obtain the following result for the existence of the periodic solution (4.1) for the system (3.1). The result is not intuitive and not expected that the delay induces many periodic solutions from the non-delay system (2.1).

Theorem 4.1 For each $\tau>0$, the Eq. (4.2) has infinitely many roots, which are countable. Thus the system (3.1) has infinitely many periodic solutions, which are countable, of the form (4.1).

\section{Analysis of the characteristic equation for the stability of the periodic solutions}

To analyze stability of the periodic solution obtained in Section 3, we study a system of a delay differential equation and an integral equation, employing the principle of linearized stability for the coupled systems of renewal equations and delay differential equations [4]. 


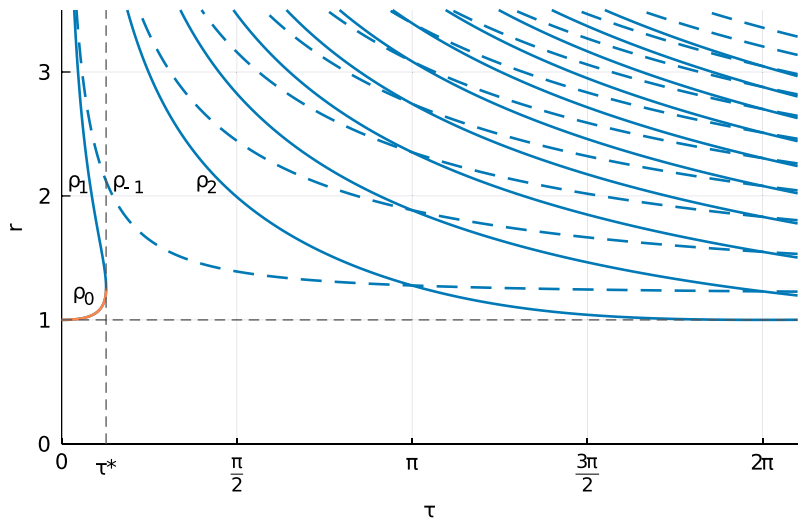

Fig. 6 Branches of the periodic solutions in $(\tau, r)$ plane. The branch $\rho_{0}$ emerges from $r=1$ at $\tau=0$ and the branch $\rho_{1}$ emerges from $r=+\infty$ at $\tau=0$. Those branches $\rho_{0}$ and $\rho_{1}$ meet at $\tau=\tau^{*}$. The branches $\rho_{j} j \in \mathbb{Z} \backslash\{0\}$ appear from $+\infty$ at $\tau=0$ and exist for $\tau>0$. Dashed curve denotes the periodic solutions with $\omega_{j}<0$ (clockwise periodic solutions)

Let $v(t)=\theta^{\prime}(t)$. From the system (3.1), we get the following system of a delay differential equation and a renewal equation

$$
\begin{gathered}
r^{\prime}(t)=r(t)\left(1-r(t) r(t-\tau) \cos \int_{t-\tau}^{t} v(s) d s\right), \\
v(t)=1+r(t) r(t-\tau) \sin \int_{t-\tau}^{t} v(s) d s .
\end{gathered}
$$

The system (5.1) has equilibria

$$
(r(t), v(t))=\left(\rho_{j}, \omega_{j}\right), j \in \mathbb{N}
$$

corresponding to the periodic solutions

$$
(r(t), \theta(t))=\left(\rho_{j}, \omega_{j} t\right), j \in \mathbb{N}
$$

of the system (3.1), given as in Sect. 3.

In the Appendix A, by linearization of the system (5.1) at the equilibrium (5.2), we obtain the following characteristic equation

$$
0=\lambda+2 e^{-\lambda \tau}-\rho^{4} \int_{0}^{2 \tau} e^{-\lambda s} d s, \lambda \in \mathbb{C},
$$

with $\rho=\rho_{j}, j \in \mathbb{N}$. Note that we have a family of the characteristic equations (5.3) which are indexed by the equilibrium about which we linearize.

Define $\eta:=\lambda \tau$. Then, from (5.3), we obtain the following equation 


$$
0=f(\eta), \eta \in \mathbb{C}
$$

where $f: \mathbb{C} \rightarrow \mathbb{C}$ defined as

$$
f(\eta):=\eta+2 \tau e^{-\eta}-\rho^{4} \tau^{2} \int_{0}^{2} e^{-\eta s} d s, \eta \in \mathbb{C} .
$$

We study (5.4) to analyze the distribution of the roots of (5.3) with respect to the imaginary axis. First let us study the existence of real roots for the characteristic equation.

Lemma 5.1 For each $j \in \mathbb{N}$, the characteristic equation (5.4) has

1. a unique negative real root if $\rho_{j}^{4} \tau<1$,

2. a root $\lambda=0$ if $\rho_{j}^{4} \tau=1$, and

3. a unique positive real root if $\rho_{j}^{4} \tau>1$.

Proof Consider the function $f(\eta)$ for $\eta \in \mathbb{R}$. Note that $f(0)=2 \tau\left(1-\rho^{4} \tau\right)$. Since it follows that

$$
\lim _{\eta \rightarrow-\infty} e^{\eta} \int_{0}^{2} e^{-\eta s} d s=\lim _{\eta \rightarrow-\infty} \frac{e^{\eta}-e^{-\eta}}{\eta}=\infty
$$

one obtains

$$
\lim _{\eta \rightarrow \infty} f(\eta)=\infty, \lim _{\eta \rightarrow-\infty} f(\eta)=-\infty
$$

Since $f$ is a continuous function, if $\rho_{j}^{4} \tau<1$ then there is a negative real root and if $\rho_{j}^{4} \tau>1$ then there is a positive real root. We also see that if $\rho_{j}^{4} \tau=1$ then there exists a root 0 for the function $f$.

For the uniqueness of the root, we study the equation $0=\eta f(\eta)$. Here

$$
\begin{aligned}
\eta f(\eta) & =\eta^{2}+2 \tau e^{-\eta} \eta-\rho^{4} \tau^{2}\left(1-e^{-2 \eta}\right) \\
& =\left(\eta+\tau e^{-\eta}\right)^{2}-\tau^{2} e^{-2 \eta}-\rho^{4} \tau^{2}\left(1-e^{-2 \eta}\right) .
\end{aligned}
$$

Let us define

$$
\begin{aligned}
& g_{1}(\eta)=\left(\eta+\tau e^{-\eta}\right)^{2}, \\
& g_{2}(\eta)=\tau^{2} e^{-2 \eta}+\rho^{4} \tau^{2}\left(1-e^{-2 \eta}\right) .
\end{aligned}
$$

We consider an intersection of $g_{1}$ and $g_{2}$. It is easy to see that $g_{1}(0)=g_{2}(0)=\tau^{2}$. We compute 


$$
\begin{aligned}
& g_{1}^{\prime}(\eta)=2\left(\eta+\tau e^{-\eta}\right)\left(1-\tau e^{-\eta}\right), \\
& g_{2}^{\prime}(\eta)=2 \tau^{2}\left(\rho^{4}-1\right) e^{-2 \eta}>0, \\
& g_{2}^{\prime \prime}(\eta)=-4 \tau^{2}\left(\rho^{4}-1\right)<0 .
\end{aligned}
$$

Therefore, one sees that $g_{1}$ is a downward-convex function (attaining minimum at $\eta=\log \tau)$ and $g_{2}$ is upward-convex function. Hence, the intersection of the functions $g_{1}$ and $g_{2}$ except 0 is unique. Thus we obtain the conclusion.

For each $j \in \mathbb{N}$ we obtain the estimation of $\rho_{j}^{4} \tau$ with respect to 1 as follows.

Lemma 5.2 The following statements are true.

- It holds $\rho_{0}^{4} \tau<1$ and $\rho_{1}^{4} \tau>1$ for $\tau<\tau^{*}$ and $\rho_{0}^{4} \tau=\rho_{1}^{4} \tau=1$ for $\tau=\tau^{*}$, and

- it holds $\rho_{j}^{4} \tau>1(j \in \mathbb{N} \backslash\{0,1\})$ for $\tau>0$.

Proof For any $j \in \mathbb{Z}$, from the condition (4.4),

$$
\rho_{j}^{4}=1+\tan ^{2} \omega_{j} \tau=\frac{1}{\cos ^{2} \omega_{j} \tau} .
$$

Thus

$$
\rho_{j}^{4} \tau=\left.\frac{d}{d \omega} \tan (\omega \tau)\right|_{\omega=\omega_{j}}
$$

holds. One can see that

$$
\left.\frac{d}{d \omega} \tan (\omega \tau)\right|_{\omega=\omega_{0}}<1<\left.\frac{d}{d \omega} \tan (\omega \tau)\right|_{\omega=\omega_{1}}
$$

for $\tau<\tau^{*}$ and

$$
\left.\frac{d}{d \omega} \tan (\omega \tau)\right|_{\omega=\omega_{0}}=1=\left.\frac{d}{d \omega} \tan (\omega \tau)\right|_{\omega=\omega_{1}}
$$

for $\tau=\tau^{*}$. Thus we obtain the first statement. For $j \in \mathbb{Z} \backslash\{0,1\}$ it is clear that

$$
\rho_{j}^{4} \tau=\left.\frac{d}{d \omega} \tan (\omega \tau)\right|_{\omega=\omega_{j}}>1
$$

Thus we obtain the conclusion.

From Lemmas 5.1 and 5.2 and the principle of linearized stability [4], we obtain the following result concerning instability of the periodic solutions.

Theorem 5.1 The periodic solution of the form (4.1) for $j=1$ is unstable for $\tau<\tau^{*}$. The periodic solution of the form (4.1) for $j \in \mathbb{Z} \backslash\{0,1\}$ is unstable. 
Let us consider stability of the periodic solution of the form (4.1) for $j=0$. For $j=0$ the characteristic equation (5.4) may have an imaginary root with positive real part. Thus so far we cannot determine stability of the periodic solution from Lemmas 5.1 and 5.2. Below we exclude this possibility to conclude that the periodic solution of the form (4.1) for $j=0$ is asymptotically stable for $\tau<\tau^{*}$.

First let us show that there is a compact region in the complex plane for the existence of a root with positive real part.

Lemma 5.3 Let $j=0$. Suppose that the characteristic Eq. (5.4) has a root $\eta$ with $\operatorname{Re} \eta>0$. Then every root $\eta$ with $\operatorname{Re} \eta>0$ satisfies

$$
|\eta| \leq 2 \tau+2 \rho_{0}^{4} \tau^{2} \frac{1}{|\eta|} .
$$

Thus for any $\varepsilon>0$ there exists $\delta>0$ such that $\tau<\delta$ implies $|\eta|<\varepsilon$.

Proof Consider the Eq. (5.4) for $\eta$ such that $\operatorname{Re} \eta>0$. We have

$$
\int_{0}^{2} e^{-\eta s} d s=\frac{1-e^{-2 \eta}}{\eta} .
$$

Thus from the characteristic equation (5.4), we obtain

$$
\eta=-2 \tau e^{-\eta}+\rho_{0}^{4} \tau^{2} \frac{1-e^{-2 \eta}}{\eta} .
$$

By the straightforward estimation $\left|e^{-\eta}\right|<1$ and $\left|1-e^{-2 \eta}\right| \leq 1+\left|e^{-2 \eta}\right|<2$ using $\operatorname{Re} \eta>0$, we obtain the estimation (5.6). From the inequality (5.6), one gets

$$
|\eta| \leq \tau+\sqrt{2 \tau+\tau^{2}}
$$

from which we obtain the conclusion.

Substituting $\eta=\mu+i v,(\eta, v) \in \mathbb{R}^{2}$ into (5.4), we obtain the following two equations.

$$
\begin{aligned}
& 0=\mu+2 \tau e^{-\mu} \cos \nu-\rho_{j}^{4} \tau^{2} \int_{0}^{2} e^{-\mu s} \cos \nu s d s, \\
& 0=\nu-2 \tau e^{-\mu} \sin \nu+\rho_{j}^{4} \tau^{2} \int_{0}^{2} e^{-\mu s} \sin \nu s d s .
\end{aligned}
$$

From $(5.7 b)$ it is immediate to obtain the following lemma. We omit the proof.

Lemma 5.4 If $\eta=\mu+i v$ is a root of the characteristic Eq. (5.4), then so is its conjugate $\bar{\eta}=\mu-i v$. 
From Lemma 5.4, it is sufficient to consider the root $\eta=\mu+i v$ of the characteristic equation (5.4) with $v>0$. Our next aim is to show the following result.

Lemma 5.5 Let $j=0$. There exists $\varepsilon$ such that if $\tau<\varepsilon$ then there is no root with positive real part for the characteristic equation (5.4).

Proof Consider the branch $j=0$ for $\tau<\tau^{*}$. Suppose that there exists a root $\eta=\mu+i v$ with $\mu>0$. From Lemma 5.3, there exists $\tau$ such that $\sin v s \geq 0$ for $s \in[0,2]$. Thus, for this $\tau$, one has $\int_{0}^{2} e^{-\mu s} \sin \nu s d s \geq 0$. Furthermore, for sufficiently small $\tau$,

$$
v-2 \tau e^{-\mu} \sin v=v\left(1-2 \tau e^{-\mu} \frac{\sin v}{v}\right)>0
$$

for $v>0$. Therefore, we obtain a contradiction to (5.7). Thus we obtain the conclusion.

Lemma 5.6 Let $j=0$. The characteristic equation (5.4) does not have a purely imaginary root.

Proof Assume that there exists an imaginary root $\eta=i v, v>0$ for the characteristic equation (5.4). Then, from (5.7b), it holds that

$$
\begin{gathered}
0=2 \tau \cos v-\rho_{0}^{4} \tau^{2} \int_{0}^{2} \cos v s d s, \\
0=v-2 \tau \sin v+\rho_{0}^{4} \tau^{2} \int_{0}^{2} \sin v s d s .
\end{gathered}
$$

One can see that

$$
\begin{aligned}
& \int_{0}^{2} \cos v s d s=\frac{\sin 2 v}{v}=\frac{2 \sin v \cos v}{v} \\
& \int_{0}^{2} \sin v s d s=\frac{1-\cos 2 v}{v}=\frac{2 \sin ^{2} v}{v} .
\end{aligned}
$$

Then, from (5.8), we obtain

$$
\begin{gathered}
0=\cos v\left(1-\rho_{0}^{4} \tau \frac{\sin v}{v}\right) \\
0=v\left(1-2 \tau \frac{\sin v}{v}+2 \rho_{0}^{4} \tau^{2}\left(\frac{\sin v}{v}\right)^{2}\right) .
\end{gathered}
$$

Note that we have $\rho_{0} \geq 1$ from (4.6). Since, for any $a \in \mathbb{R}$, it holds that $1-2 \tau a+2 \tau^{2} a^{2}>0$, we see that 


$$
1-2 \tau \frac{\sin v}{v}+2 \rho_{0}^{4} \tau^{2}\left(\frac{\sin v}{v}\right)^{2} \geq 1-2 \tau \frac{\sin v}{v}+2 \tau^{2}\left(\frac{\sin v}{v}\right)^{2}>0,
$$

which is a contradiction to (5.9b). Therefore, we obtain the conclusion.

Therefore, from the application of the Rouche's theorem (see e.g. Lemma 2.8 in Chapter XI of [5]), we obtain the following conclusion.

Theorem 5.2 If $\tau<\tau^{*}$, then the periodic solution of the form (4.1) with $j=0$ is asymptotically stable and the periodic solution $j \neq 0$ is unstable. If $\tau>\tau^{*}$ then every periodic solution (4.1) is unstable.

From Theorems 4.1 and 5.2, we complete the proof of Theorem 2.2.

\section{Numerical simulations}

In this section we demonstrate numerical solutions of the delay differential equation (2.1) with the special initial conditions $x(t)=y(t)=\delta, t \in[-\tau, 0]$, where $\delta \in \mathbb{R}$.

First we fix $\tau=0.392$. For $\tau<\tau^{*} \approx 0.398284 \cdots$, we show that the periodic solution of the form (4.1) with $j=0$ is asymptotically stable (Theorem 5.2). From (4.4), we can compute the radius of the stable periodic solution as $r=\rho_{0} \approx 1.18547 \cdots$. In Fig. 7 , a trajectory of the solution for $\delta=-36$ is plotted in $(x, y)$ plane, which shows that the periodic solution with the radius $r=\rho_{0} \approx 1.18547 \cdots$ attracts the solution. Observe that there is an unstable periodic solution in the vicinity of the stable periodic solution (in Fig. 7, the trajectory of the asymptotically stable periodic solution and of the unstable periodic solution are illustrated as the dashed orange circle and as the dashed blue circle, respectively). In Fig. 8, a trajectory of the solution with the initial condition $\delta=-37$ is plotted. In this case, the solution winds around the unstable periodic solution (dashed blue circle) and then leaves and goes far away.

In Fig 9, we plot $\log r(t)$ for several initial conditions $\delta$. The numerical experiment suggests that the solution exists globally for $-36<\delta \leq 0$ and blows up for $\delta<-37$ : in short, the solution blows up for large $|\delta|$. We can numerically observe many blow-up solutions, which blow up even after $t=\tau / 2$. In this paper, we prove the existence of blow-up solutions, which blow up in the time interval $(0, \tau / 2)$. The numerical simulation suggest that many solutions blow up in finite times.

We also numerically compute the solution for $\tau>\tau^{*}$. Figure 10 shows a transient behavior of a solution. The solution stays around the origin for a while and then it blows up in a finite time. Although for $\tau=0.3985>\tau^{*}$, there does not exist periodic solution for $j=0$ and $j=1$ around the origin, those periodic solutions may indirectly affect the transient behavior of the solution. 


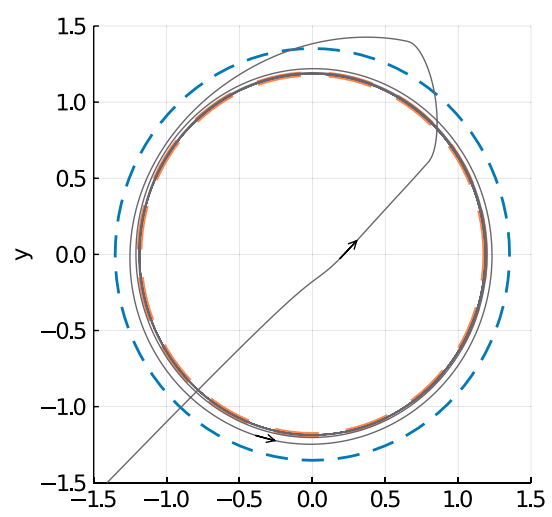

Fig. 7 Illustration of a trajectory of the solution with $\delta^{\mathrm{X}}=-36$ in $(x, y)$-plane for $\tau=0.392$. The asymptotic stable solution attracts the solution with the initial condition $\delta=-36$. The trajectory of the asymptotically stable periodic solution and of the unstable periodic solution are illustrated as the dashed orange circle and as the dashed blue circle, respectively
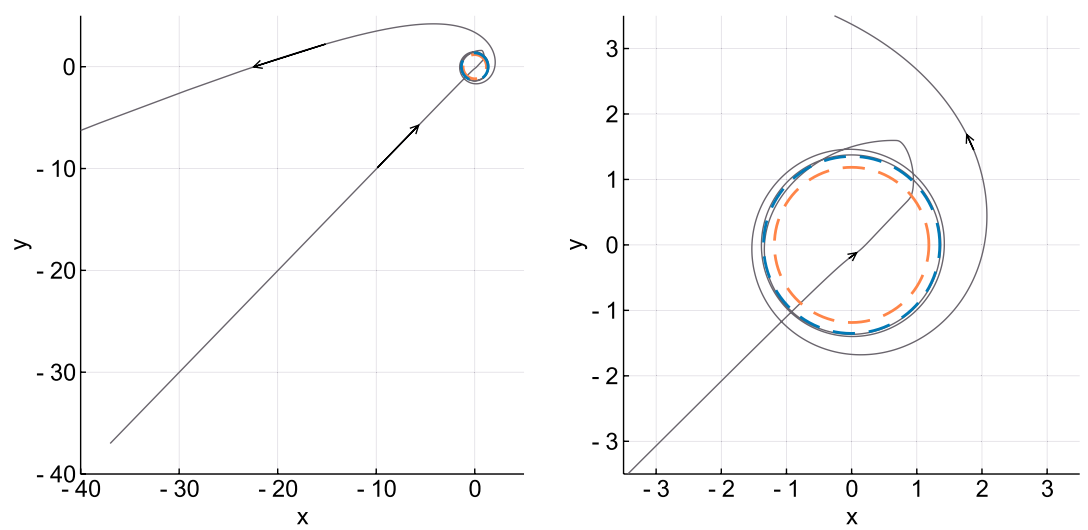

Fig. 8 Illustration of a trajectory of the solution with $\delta=-37$ in $(x, y)$-plane for $\tau=0.392$. (The right figure is close-up view of the left figure near the origin.) The solution blows up, after winding around the unstable periodic solution (dashed blue circle). The trajectory of the asymptotically stable periodic solution and of the unstable periodic solution are illustrated as the dashed orange circle and as the dashed blue circle, respectively

\section{Discussion}

In this paper we show an example of a blow-up phenomenon in a planar system of delay differential equations. In our system, the delay completely changes the system: many blow-up solutions and periodic solutions suddenly appear, no matter how small the length of the delay is. In neutral delay differential equations, it is known that arbitrary small delay can destabilize the system (see Chapter 1.7 in [9]). Here we find that arbitrary small delay can induce blow-up solutions in delay differential equations. Numerical simulations suggest that many solutions either 


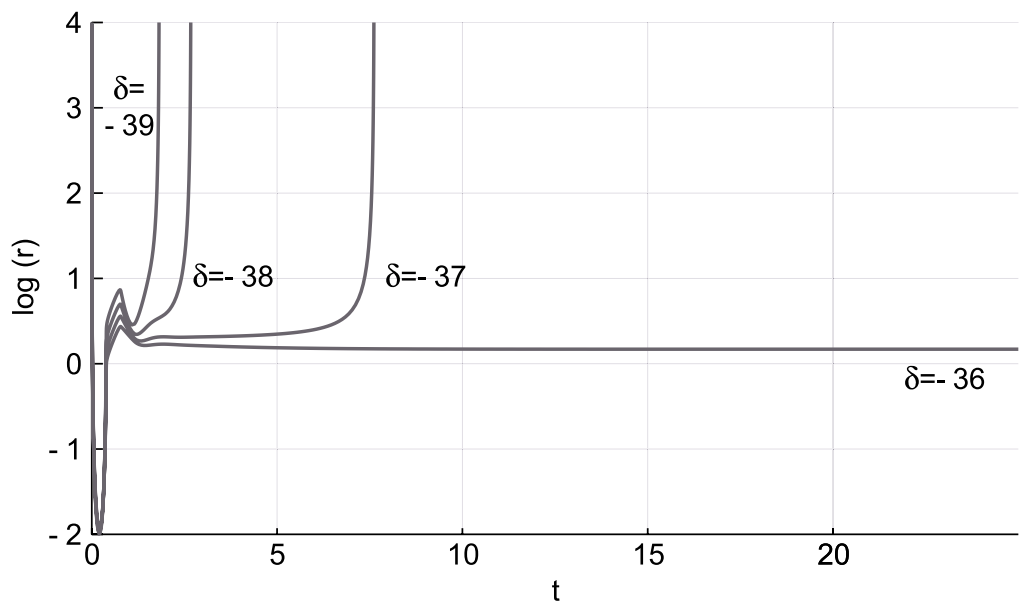

Fig. 9 We plot the growth of $\log r(t)$ for several $\delta$. Here $\tau$ is fixed $(\tau=0.392)$. The numerical simulation suggests that larger $|\delta|$ causes the solution to blow up faster

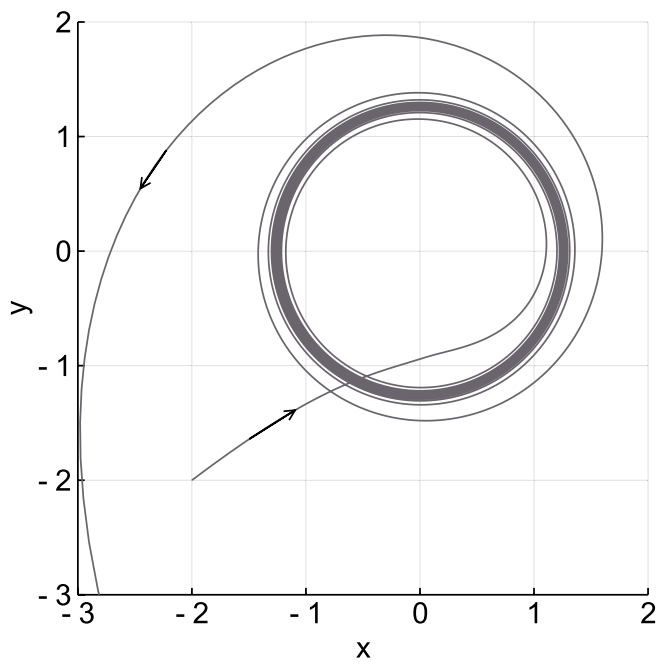

Fig. 10 Transient behavior of the solution for $\tau=0.3985>\tau^{*}$. The solution stays around the origin for a while, then it blows up in a finite time

tend to the stable periodic solution or blow up in a finite time. It is not obvious if more complicated solution behavior exists. The transient behavior observed in Fig. 10 is interesting, as it looks that the solution tries to find a stable periodic solution which does not exist in this parameter setting.

Many results concerning the existence of the blow-up solutions are available in Volterra integral equations (see $[2,3,15,16]$ and references therein). When delay 
differential equations can be formulated as Volterra integral equations, we may apply the blow-up results for Volterra integral equations to delay differential equations, see e.g. $[1,21]$ for a relation between Volterra type integral equations and integro-differential equations. However, we cannot apply the results for Volterra integral equations to a system of integral equations which is rewritten formally from our target system of delay differential equations. Since delay differential equations form an infinite dimensional dynamical system, it is also not straightforward to apply the results established in ordinary differential equations.

In the context of mathematical modelling, our example suggests that arbitrary small delay can be responsible for a drastic change of the dynamics, thus one should be careful when ignoring small delay. Other blow-up mechanisms in delay differential equations will be explored in our future work.

\section{A The characteristic equation}

Here, by linearization of the system (5.1) about the equilibrium (5.2), we derive the characteristic equation (5.3), which characterizes stability of the periodic solutions. Fixing $j \in \mathbb{Z}$, we let

$$
s_{j}(t)=r(t)-\rho_{j}, u(t)=v(t)-\omega_{j} .
$$

Then, applying the Taylor expansion, we get

$$
\begin{aligned}
& \cos \int_{t-\tau}^{t} v(s) d s=\cos \int_{t-\tau}^{t}\left(u(s)+\omega_{j}\right) d s=\cos \omega_{j} \tau-\sin \omega_{j} \tau \int_{t-\tau}^{t} u(s) d s+\cdots, \\
& \sin \int_{t-\tau}^{t} v(s) d s=\sin \int_{t-\tau}^{t}\left(u(s)+\omega_{j}\right) d s=\sin \omega_{j} \tau+\cos \omega_{j} \tau \int_{t-\tau}^{t} u(s) d s-\cdots .
\end{aligned}
$$

Ignoring the higher order terms, we obtain the following linearized equation

$$
\begin{gathered}
s^{\prime}(t)=-s(t)-s(t-\tau)+\rho_{j}^{3} \sin \omega_{j} \tau \int_{0}^{\tau} u(t-s) d s, \\
u(t)=\rho_{j} \sin \omega_{j} \tau(s(t)+s(t-\tau))+\int_{0}^{\tau} u(t-s) d s .
\end{gathered}
$$

Substituing the exponential solution $(s(t), u(t))=e^{\lambda t} \mathbf{c}$, where $\lambda \in \mathbb{C}$ and $\mathbf{c} \in \mathbb{C}^{2}$, we get the following characteristic equation

$$
\operatorname{det}\left(\left[\begin{array}{cc}
-1-e^{-\lambda \tau} & \rho_{j}^{3} \sin \omega \tau \int_{0}^{\tau} e^{-\lambda s} d s \\
\rho_{j} \sin \omega_{j} \tau\left(1+e^{-\lambda \tau}\right) & \int_{0}^{\tau} e^{-\lambda s} d s
\end{array}\right]-\left[\begin{array}{ll}
\lambda & 0 \\
0 & 1
\end{array}\right]\right)=0 .
$$

Using (4.2a), we have 


$$
\rho_{j}^{4} \sin ^{2} \omega \tau=\rho_{j}^{4}-\rho_{j}^{4} \cos ^{2} \omega \tau=\rho_{j}^{4}-1 .
$$

Therefore, the characteristic equation (A.2) becomes

$$
\left(1+e^{-\lambda \tau}+\lambda\right)\left(1-\int_{0}^{\tau} e^{-\lambda s} d s\right)-\left(\rho_{j}^{4}-1\right)\left(1+e^{-\lambda \tau}\right) \int_{0}^{\tau} e^{-\lambda s} d s=0
$$

The Eq. (A.3) can be written as

$$
\left(1+e^{-\lambda \tau}+\lambda\right)-\int_{0}^{\tau} e^{-\lambda s} d s\left(\lambda+\rho_{j}^{4}\left(1+e^{-\lambda \tau}\right)\right)=0 .
$$

Therefore, we obtain the characteristic equation (5.3) in the main text.

Acknowledgements The authors thank the referees for careful reading and invaluable comments on our manuscript.

Open Access This article is licensed under a Creative Commons Attribution 4.0 International License, which permits use, sharing, adaptation, distribution and reproduction in any medium or format, as long as you give appropriate credit to the original author(s) and the source, provide a link to the Creative Commons licence, and indicate if changes were made. The images or other third party material in this article are included in the article's Creative Commons licence, unless indicated otherwise in a credit line to the material. If material is not included in the article's Creative Commons licence and your intended use is not permitted by statutory regulation or exceeds the permitted use, you will need to obtain permission directly from the copyright holder. To view a copy of this licence, visit http://creativecommons.org/licen ses/by/4.0/.

\section{References}

1. Appleby, J.A.D., Patterson, D.D.: Blow-up and superexponential growth in superlinear Volterra equations. Disc. Contain. Dyn. Syst. 38(8), 3993-4017 (2017)

2. Bandle, C., Brunner, H.: Blowup in diffusion equations: a survey. J. Comput. Appl. Math. 97(1-2), 3-22 (1998)

3. Brunner, H., Yang, Z.W.: Blow-up behavior of Hammerstein-type Volterra integral equations. J. Int. Equ. Appl. 24(4), 487-512 (2012)

4. Diekmann, O., Getto, Ph, Gyllenberg, M.: Stability and bifurcation analysis of Volterra functional equations in the light of suns and stars. SIAM J. Math. Anal. 39(4), 1023-1069 (2008)

5. Diekmann, O., van Gils, S.A., Verduyn Lunel, S.M., Walther, H.O.: Delay Equations: Functional-, Complex-, and Nonlinear Analysis, Applied Mathematical Sciences (Vol. 110), Springer, New York (1995)

6. Elias, U., Gingold, H.: Critical points at infinity and blow up of solutions of autonomous polynomial differential systems via compactification. J. Math. Anal. Appl. 318(1), 305-322 (2006)

7. Erneux, T.: Applied Delay Differential Equations. Springer, New York (2009)

8. Ezzinbi, K., Jazar, M.: Blow-up results for some nonlinear delay differential equations. Positivity 10, 329-341 (2006)

9. Hale, J.K., Verduyn Lunel, S.M.: Introduction to Functional Differential Equations. Springer, New York (1993)

10. Hirata, D.: Blow-up for a class of semilinear integro-differential equations of parabolic type. Math. Methods. Appl. Sci. 22(13), 1087-1100 (1999) 
11. Hirota, C., Ozawa, K.: Numerical method of estimating the blow-up time and rate of the solution of ordinary differential equations: an application to the blow-up problems of partial differential equations. J. Comput. Appl. Math. 193(2), 614-637 (2006)

12. Kuznetsov, Y.A.: Elements of Applied Bifurcation Theory. Springer, New York (1998)

13. Ma, J.: Blow-up solutions of nonlinear Volterra integro-differential equations. Math. Comput. Model. 54(11-12), 2551-2559 (2011)

14. Matsue, K.: On blow-up solutions of differential equations with Poincaré-type compactifications. SIAM J. Appl. Dyn. Syst. 17(3), 2249-2288 (2018)

15. Mydlarczyk, W.: A condition for finite blow-up time for a Volterra integral equation. J. Math. Anal. Appl. 181(1), 248-253 (1994)

16. Roberts, C.A.: Recent results on blow-up and quenching for nonlinear Volterra equations. J. Comput. Appl. Math. 205(2), 736-743 (2007)

17. Smith, H.: An Introduction to Delay Differential Equations with Applications to the Life Sciences. Springer, New York (2011)

18. Souplet, P.: Blow-up in nonlocal reaction-diffusion equations. SIAM J. Math. Anal. 29(6), 1301$1334(1998)$

19. Straughan, B.: Explosive Instabilities in Mechanics. Springer, New York (2012)

20. Takayasu, A., Matsue, K., Sasaki, T., Tanaka, K., Mizuguchi, M., Oishi, S.: Numerical validation of blow-up solutions of ordinary differential equations. J. Comput. Appl. Math. 314, 10-29 (2017)

21. Yang, Z., Brunner, H.: Blow-up behavior of Hammerstein-type delay Volterra integral equations. Front. Math. Chin. 8, 261-280 (2013)

22. Yang, Z., Tang, T., Zhang, J.: Blowup of Volterra integro-differential equations and applications to semi-linear Volterra diffusion equations. Num. Math. 10(4), 737-759 (2017)

23. Zhou, Y.C., Yang, Z.W., Zhang, H.Y., Wang, Y.: Theoretical analysis for blow-up behaviors of differential equations with piecewise constant arguments. Appl. Math. Comp. 274, 353-361 (2016)

Publisher's Note Springer Nature remains neutral with regard to jurisdictional claims in published maps and institutional affiliations. 